\title{
Habenular expression of rare missense variants of the $\beta 4$ nicotinic receptor subunit alters nicotine consumption
}

\author{
Marta A. Ślimak', Jessica L. Ables ${ }^{2}$, Silke Frahm ${ }^{1}$, Beatriz Antolin-Fontes ${ }^{1,2}$, Julio Santos-Torres ${ }^{1}$, \\ Milena Moretti ${ }^{3}$, Cecilia Gotti ${ }^{3}$ and Inés Ibañez-Tallon ${ }^{1,2 *}$ \\ ${ }^{1}$ Molecular Neurobiology Group, Max Delbrück Center for Molecular Medicine, Berlin, Germany \\ ${ }^{2}$ Laboratory of Molecular Biology, The Rockefeller University, New York, NY, USA \\ ${ }^{3}$ Consiglio Nazionale delle Ricerche Institute of Neuroscience and Biometra Department, University of Milan, Milan, Italy
}

Edited by:

Ramiro Salas, Baylor College of

Medicine, USA

\section{Reviewed by:}

David L. Molfese, Baylor College of

Medicine, USA

Christie D. Fowler, The Scripps

Research Institute, USA

*Correspondence:

Inés Ibañez-Tallon, Laboratory of Molecular Biology, The Rockefeller University, 1230 York Avenue, New York, NY 10065, USA

e-mail: iibanez@rockefeller.edu
The CHRNA5-CHRNA3-CHRNB4 gene cluster, encoding the $\alpha 5, \alpha 3$, and $\beta 4$ nicotinic acetylcholine receptor (nAChR) subunits, has been linked to nicotine dependence. The habenulo-interpeduncular ( $\mathrm{Hb}$-IPN) tract is particularly enriched in $\alpha 3 \beta 4 \mathrm{nAChRs}$. We recently showed that modulation of these receptors in the medial habenula $(\mathrm{MHb})$ in mice altered nicotine consumption. Given that $\beta 4$ is rate-limiting for receptor activity and that single nucleotide polymorphisms (SNPs) in CHRNB4 have been linked to altered risk of nicotine dependence in humans, we were interested in determining the contribution of allelic variants of $\beta 4$ to nicotine receptor activity in the MHb. We screened for missense SNPs that had allele frequencies $>0.0005$ and introduced the corresponding substitutions in Chrnb4. Fourteen variants were analyzed by co-expression with $\alpha 3$. We found that $\beta 4 \mathrm{~A} 90 \mathrm{I}$ and $\beta 4 \mathrm{~T} 374 \mathrm{I}$ variants, previously shown to associate with reduced risk of smoking, and an additional variant $\beta 4 D 447 Y$, significantly increased nicotine-evoked current amplitudes, while $\beta 4 \mathrm{R} 348 \mathrm{C}$, the mutation most frequently encountered in sporadic amyotrophic lateral sclerosis (sALS), showed reduced nicotine currents. We employed lentiviruses to express $\beta 4$ or $\beta 4$ variants in the $\mathrm{MHb}$. Immunoprecipitation studies confirmed that $\beta 4$ lentiviral-mediated expression leads to specific upregulation of $\alpha 3 \beta 4$ but not $\beta 2 \mathrm{nAChRs}$ in the Mhb. Mice injected with the $\beta 4$-containing virus showed pronounced aversion to nicotine as previously observed in transgenic Tabac mice overexpressing Chrnb4 at endogenous sites including the $\mathrm{MHb}$. Habenular expression of the $\beta 4$ gain-of-function allele T374I also resulted in strong aversion, while transduction with the $\beta 4$ loss-of function allele R348C failed to induce nicotine aversion. Altogether, these data confirm the critical role of habenular $\beta 4$ in nicotine consumption, and identify specific SNPs in CHRNB4 that modify nicotine-elicited currents and alter nicotine consumption in mice.

Keywords: medial habenula, nicotine consumption, SNP, lentivirus transduction, electrophysiological recordings, smoking dependence

\section{INTRODUCTION}

As the leading preventable cause of cancer and death, nicotine use and dependence has been the subject of a multitude of genetic studies in the past decade. By far, the strongest and most replicable evidence exists for a role of the CHRNA5-A3-B4 gene cluster on chromosome 15q25 in nicotine addiction, which encodes three subunits of nicotinic acetylcholine receptors (nAChR). Two single nucleotide polymorphisms (SNPs) in particular, rs16969968 in CHRNA5 and rs1051730 in CHRNA3, have been linked to smoking-related behaviors in multiple studies (Bierut et al., 2007; Saccone et al., 2007; Thorgeirsson et al., 2008). Though not as strongly linked, the $\beta 4$ subunit has been nominally associated with smoking quantity in several studies (Saccone et al., 2009; Harari et al., 2012). Variants located upstream of CHRNB4 affect the age at which individuals transition to daily and habitual smoking (Kapoor et al., 2012), while another study found that SNP rs12914008 in CHRNB4 is associated with less abstinence over time (Sarginson et al., 2011). Rare missense variants in CHRNB4 (T375I and T91I) and in CHRNA3 (R37H) are associated with lower risk for nicotine dependence and fewer cigarettes per day (Haller et al., 2012).

Despite the preponderance of evidence linking the CHRNA5A3-B4 gene cluster to nicotine-related diseases, very little evidence exists as to how mutations in these receptors alter their function. Cumulative data from rodent models suggest that CHRNA5 and CHRNB4, particularly in the medial habenula (MHb)interpeduncular (IPN) circuit, mediate the aversive properties of nicotine (Salas et al., 2009; Fowler et al., 2011; Frahm et al., 2011) and withdrawal (Salas et al., 2009; Gorlich et al., 2013). For example, animals lacking the $\alpha 5 \mathrm{nAChR}$ subunit self-administer more nicotine (Fowler et al., 2011) and do not display somatic signs of nicotine withdrawal (Salas et al., 2009). Restoration of CHRNA5 to the MHb leads to normalization of nicotine consumption, i.e., the mice reach a plateau in administration, 
suggesting that it is the aversive aspect that limits intake (Fowler et al., 2011). Overexpression of the entire human gene cluster in mice leads to increased sensitivity to nicotine with higher activation of the MHb and reduced activation of the VTA (Gallego et al., 2012). We previously demonstrated that overexpression of the $\beta 4 \mathrm{nAChR}$ subunit in the Tabac transgenic mouse model is sufficient to increase sensitivity to the aversive properties of nicotine and decrease consumption. Overexpression of $\beta 4$ also leads to increased surface expression of $\alpha 3$, as $\beta 4$ is the rate-limiting subunit for assembly (Frahm et al., 2011). In particular we previously showed that $\beta 4$ is rate-limiting for $\alpha 3 \beta 4$ activity since when overexpressed it increases the density of $\alpha 3 \beta 4$ receptors at the plasma membrane, as well as potentiates $\alpha 3 \beta 4$ currents in vitro and in a transgenic mouse model (Frahm et al., 2011). The ability of $\beta 4$ to enhance nicotine-evoked currents depends on a unique single residue S435, which does not exist in other $\beta \mathrm{nAChR}$ subunits and can confer this capability to $\beta 2$ when introduced in the corresponding arginine residue (Frahm et al., 2011).

This critical residue (S435) in $\beta 4$ is located within the membrane-associated stretch in the intracellular vestibule of the receptor (Frahm et al., 2011). Sequence alignments identified other SNPs mapping to the intracellular vestibule, one of them being the most common SNP associated with nicotine use, rs16969968, leading to an amino acid substitution (D398N) in the $\alpha 5$ subunit. Functional analysis of this SNP in $\alpha 5$ demonstrated that mutation of this residue within the highly electrostatically charged intracellular vestibule of the $\mathrm{nAChR}$ significantly reduces nicotine-evoked currents. Behaviorally, this genetic variant had profound effects: Tabac mice with strong aversion to nicotine due to increased expression of $\beta 4$, reverted to nicotine preference upon viral-mediated expression of the $\alpha 5 \mathrm{D} 398 \mathrm{~N}$ variant (Frahm et al., 2011). Given that several SNPs in $\beta 4$ have been identified in humans, particularly in the intracellular vestibular region, we were interested in determining their contribution to $\alpha 3 \beta 4$ receptor function. Based on reported validation studies and heterozygosity rates, we selected 14 out of 67 missense SNPs identified in the coding region of CHRNB4. Expression of these missense $\beta 4$ variants in oocytes indicated that amino acid substitutions corresponding to 12 of these 14 SNPs led to changes in the electrophysiological properties of the channel (Liang et al., 2005; Haller et al., 2012). We further analyzed four of these SNPS in hippocampal neurons, and found that the two variants most associated with decreased risk of nicotine dependence, A90I and T374I (Haller et al., 2012), and a third variant D447Y, augmented nicotine-mediated $\alpha 3 \beta 4 \mathrm{nAChR}$ currents, while the fourth analyzed SNP, previously associated with sporadic amyotrophic lateral sclerosis (sALS) (Moriconi et al., 2011), reduced nicotine currents. Mice injected in the MHb with lentiviruses carrying the wild-type $\beta 4$ subunit or $\beta 4$ rare missense variants, showed aversion or preference to nicotine, depending on whether the variant increased or decreased nicotine currents. These results indicate that nicotine consumption is regulated by $\beta 4^{*} \mathrm{nAChR}$ activity in the $\mathrm{MHb}$.

\section{MATERIALS AND METHODS ANIMALS}

Transgenic Tabac mice were obtained from GENSAT (Gong et al., 2003) and backcrossed to a C57BL/6 background. All transgenic animals used for experiments were hemizygous. C57BL/6 male mice were purchased from Charles River (Germany) and allowed to habituate to the facility for 1 week before use in lentiviral experiments. Mice were housed with ad libitum access to food and water in a room air conditioned at $22-23^{\circ} \mathrm{C}$ with a standard $12 \mathrm{~h}$ light/dark cycle, with a maximum of five animals per cage. All procedures were in accordance with ethical guidelines laid down by the local governing body and approved by the Landesant Für Gesundheit und Soziales, Berlin and the Institutional animal use and care committee IACUC, New York).

\section{TWO-ELECTRODE VOLTAGE-CLAMP RECORDINGS OF XENOPUS LAEVIS OOCYTES}

cDNA encoding for the mouse $\alpha 3$ and $\beta 4 \mathrm{nAChR}$ subunit was subcloned into the pCS2A plasmid for oocyte expression. Sitedirected mutations corresponding to the selected SNPs were introduced in the $\beta 4 \mathrm{nAChR}$ subunit using QuikChange SiteDirected mutagenesis kit (Stratagene, catalog \# 200519). RNA transcripts were prepared using the mMESSAGE mMACHINE kit (Ambion, catalog \# AM1344M) as described (Ibanez-Tallon et al., 2004). Oocytes were surgically extracted and prepared as described (Sturzebecher et al., 2010). For electrophysiological recordings of the $14 \beta 4$ variants shown in Figure 1, each oocyte was injected with $20 \mathrm{nl}$ of a cRNA mix containing $1 \mathrm{ng}$ of $\alpha 3+1 \mathrm{ng}$ of $\beta 4$ ( $\beta 4 \mathrm{wt}$ or each of the 14 indicated $\beta 4$ variants).

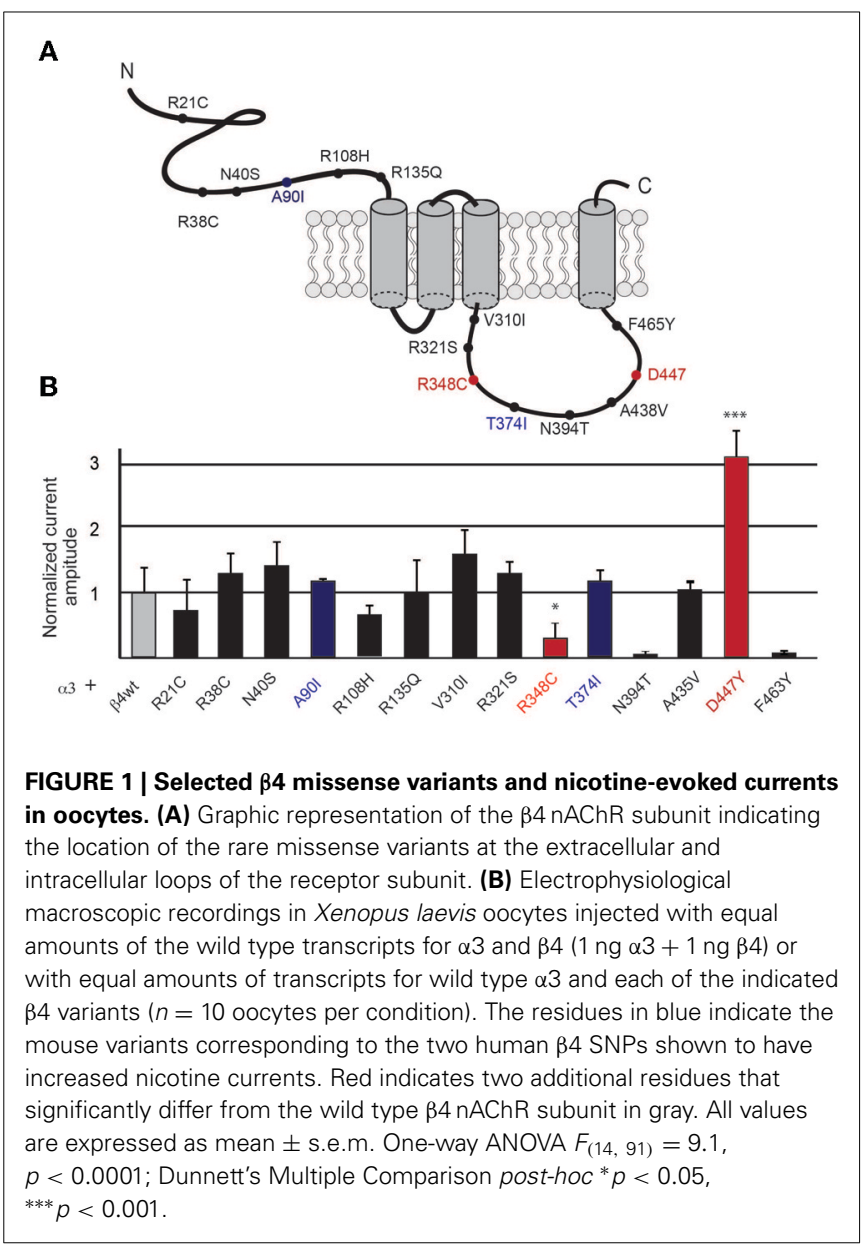


For electrophysiological recordings of the $\beta 4$ variants A90I, T374I, D447Y, and R348C shown in Figure 2, each oocyte was injected at $1: 1$ and 1:10 ratios, with either $1 \mathrm{ng}$ of $\alpha 3+1 \mathrm{ng}$ of $\beta 4$ (wt or variants): or $1 \mathrm{ng}$ of $\alpha 3+10 \mathrm{ng}$ of $\beta 4$ (wt or variants). Macroscopic currents were recorded 4 days after injection with a GeneClamp $500 \mathrm{~B}$ amplifier (Axon Instruments) using a two-electrode voltage clamp with active ground configuration as described in Frahm et al. (2011). Nicotine tartrate salt (Sigma-Aldrich, catalog \# N5260) was applied at the concentration of $1 \mathrm{mM}$. Mean fold current increase was evaluated by dividing peak amplitudes of 5-10 single oocytes expressing receptors containing each of the polymorphic variants of $\beta 4 \mathrm{nAChR}$ by peak amplitudes of the ones carrying native $\beta 4 \mathrm{nAChR}$ at $1: 1$ and $1: 10$ ratio.

\section{LENTIVIRUS PRODUCTION}

Recombinant lentiviral vectors were prepared using transient transfection of HEK293T cells as described in (Auer et al., 2010).

\section{WHOLE-CELL PATCH CLAMP RECORDINGS IN PRIMARY HIPPOCAMPAL NEURONS}

Dissociated hippocampal cultures were prepared from embryonic day 19 rat embryos and prepared as described in (Auer et al., 2010). After 9 days in culture, neurons were infected with lentiviruses carrying the $\alpha 3$ and one of the variants of $\beta 4 \mathrm{nAChR}$ subunit. Three to four days after lentivirus infection, nicotineelicited currents were analyzed by whole-cell patch-clamp recordings. The internal pipette solution contained $(\mathrm{mM}): 130 \mathrm{KCl}$, $2 \mathrm{MgCl}_{2}, 0.5 \mathrm{CaCl}_{2}, 5$ EGTA, 10 HEPES, pH 7.3, osmolarity 280 (resistance 5-7 M $\Omega$ ). $30 \mu \mathrm{M}$ nicotine tartrate was locally applied

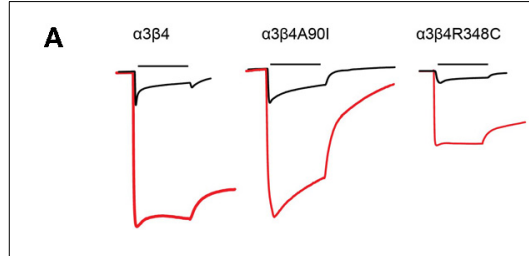

$\mathbf{B}$

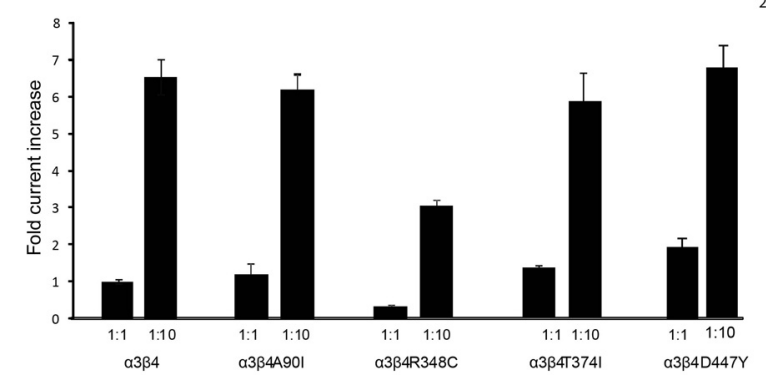

FIGURE 2 | Increased currents by overexpression of $\boldsymbol{\beta} 4$ variants. (A) Representative traces of two-electrode-voltage-clamp recordings in oocytes injected with either equal amounts of $\alpha 3$ and $\beta 4$ transcripts ( $1 \mathrm{ng}$ $\alpha 3+1$ ng $\beta 4,1: 1$ ratio; black traces) or with 10 times more of the $\beta 4$ transcript (1 ng $\alpha 3+10 \mathrm{ng} \beta 4,1: 10$ ratio; red traces). Horizontal line above each trace indicates nicotine $(1 \mathrm{mM})$ application. (B) Bar graph indicates the fold increase of the current amplitude elicited by $1 \mathrm{mM}$ nicotine with respect to a3b4 wt oocytes injected at 1:1 ( $n=10$ oocytes per condition) for oocytes injected with $1: 1$ or $1: 10$ rations of the indicated $\beta 4$ variants A90I, T374I, D447Y, and R348C. All values are expressed as mean \pm s.e.m.
(50 ms, 18-10 psi) with a pressure device (PR-10, ALA Scientific Instruments) connected to a focal perfusion system (VM4, ALA Scientific Instruments) controlled with a trigger interface (TIB $14 \mathrm{~S}, \mathrm{HEKA})$. The pipette was moved within $15-20 \mu \mathrm{m}$ of the recorded cell with a motorized micromanipulator (LN mini 25, control system SM-5, Luigs and Neumann) for drug application and retracted after the end of the puff to minimize desensitization. Currents were recorded with a HEKA amplifier (EPC 10) using PatchMaster software (V2.20, HEKA), and analyzed with FitMaster software (V2.3, HEKA). Membrane potential was held at $-70 \mathrm{mV}$.

\section{STEREOTACTIC VIRAL INJECTIONS IN THE MHb}

Stereotactic injections were performed as described in Frahm et al. (2011). Briefly, $1 \mu \mathrm{l}$ of concentrated lentivirus $(2-5 \times$ $10^{8} \mathrm{TU} / \mathrm{ml}$ ) was injected bilaterally into the MHb of 8 week-old $\mathrm{C} 57 \mathrm{Bl} / 6$ male mice at each of the following coordinates from bregma (Franklin and Paxinos, 2008): antero-posterior -1.40 and -1.74 , lateral \pm 0.33 , dorso-ventral (from skull level) -2.7 and -2.74 . Behavioral experiments started 2 weeks after injection with either the wild type $\beta 4$-carrying lentivirus pFU- $\beta 4$-IRESEGFP or virus carrying one of the $\beta 4$-point mutants. Expression of eGFP using the same pFU lentiviral vectors has been shown after just 1 week (Dittgen et al., 2004). Immunoprecipitation assays or sacrifice followed by transcardial perfusion with $4 \%$ paraformaldehyde in PBS were done 4 weeks post-injection. Verification of the injection site for each animal in the behavioral assays was performed on $40 \mu \mathrm{m}$ coronal brain sections immunostained with rabbit polyclonal anti-GFP antibody (Invitrogen, catalog \# A11122) diluted 1:1000 as previously described (Frahm et al., 2011; Gorlich et al., 2013). Animals with lentiviral injection placement outside the $\mathrm{MHb}$ were excluded from behavioral analysis, leaving 6-8 animals per group.

\section{IMMUNOPRECIPITATION OF $\left[{ }^{125}\right.$ I] EPIBATIDINE-LABELED RECEPTORS BY SUBUNIT-SPECIFIC ANTIBODIES}

Four weeks post-injection, habenula were dissected from C57BL/ 6 mice injected with either the $\beta 4$-carrying lentivirus pFU- $\beta 4$-IRES-EGFP or control virus $\mathrm{pFU}-\mathrm{PE}$, and were processed as previously described (Grady et al., 2009). Briefly, 2\% Triton X-100 habenula extracts were preincubated with $2 \mu \mathrm{M}$ $\alpha$ Bgtx (Tocris, Bristol, UK catalog \# 2133), labeled with $0.5 \mathrm{nM}$ $\left[{ }^{125} \mathbf{I}\right]$ Epibatidine (specific activity $2200 \mathrm{Ci} / \mathrm{mmol}$, Perkin Elmer catalog \#NEX358) and incubated overnight with a saturating concentration of affinity-purified anti-subunit $\alpha 3, \beta 4$, and $\beta 2$ specific polyclonal IgG produced and characterized by Grady et al. (2009). These polyclonal antibodies [supplementary Table of Grady et al. (2009)] were produced against the following peptide sequences TRPTSTEEDAPKTRNFYGAELSNLNC in the cytosolic loop of mouse $\alpha 3$, VSSHTAGLPRDARLRSSGRFREDLQEALEG in the cytosolic loop of rat $\beta 4$, and RQREREGAGALFFREAPGADSCTY in the cytosolic loop of human $\beta 2$. As described in (Grady et al., 2009 ), the specificity of the anti $\beta 4$ and anti $\beta 2$ antibodies, was tested by immunoprecipitation using ${ }^{3} \mathrm{H}$ - or ${ }^{125} \mathrm{I}$-Epibatidine labeled receptors obtained from tissues of wt and $\beta 4$ and $\beta 2$ $\mathrm{KO}$ mice respectively; specific immunopreciptation was obtained in wt but not in the respective $\mathrm{KO}$ mice. The anti- $\alpha 3$ antibody 
specificity was determined by specific immunoprecipitation of anti- $\alpha 3$ antibodies only in HEK cells transfected with the $\alpha 3 \beta 4$ or $\alpha 3 \beta 2$ subtypes and not in HEK cells transfected with the human $\alpha 2 \beta 2, \alpha 2 \beta 4, \alpha 4 \beta 2$, and $\alpha 4 \beta 4$ subtypes. After incubating with the specific polyclonal antibodies, the immunoprecipitated fractions from habenula extracts were recovered by incubating the samples with beads containing bound anti-rabbit goat IgG (Technogenetics). The level of antibody immunoprecipitation was expressed as femtomoles of immunoprecipitated receptors per milligram of protein.

\section{TWO-BOTTLE NICOTINE CONSUMPTION}

The two-bottle drinking test was used to measure the consumption to nicotine relative to water, as described in Meliska et al. (1995), Robinson et al. (1996), Butt et al. (2005), Glatt et al. (2009). We used a moderate dose of $50 \mu \mathrm{g} / \mathrm{ml}$ nicotine (Matta et al., 2007) since at this dose we previously observed a large difference in nicotine consumption between wild type and Tabac mice (Frahm et al., 2011). Adult male Tabac mice or lentiviral injected C57BL/6 mice were single-housed and tested in their home cages. For the first 3 days, nicotine naïve mice were presented with two bottles of $2 \%$ saccharin in tap water for acclimation to the test conditions. After 3 days, the volume consumed from each bottle was recorded to determine any baseline side preference. The difference in volume before and after 3 day consumption was measured by weighting the cage bottles $(0.5 \mathrm{ml}$ is the smallest difference that can be measured by weighting). After the acclimation period, the solution in one of the bottles was replaced with nicotine tartrate $(50 \mu \mathrm{g} / \mathrm{ml})$ in $2 \%$ saccharin in tap water, and the volume drunk from each bottle was measured after 3 days. To account for side-bias, nicotine was presented on the side that was less preferred. Bottles were equipped with sipper tubes with ball bearings to prevent volume loss by leakage. Percent nicotine consumption was expressed as a ratio of the volume of nicotine solution consumed divided by the total fluid intake $(\mathrm{ml}$ nicotine $/ \mathrm{ml}$ total $\times 100)$.

\section{STATISTICAL ANALYSIS}

All data are presented as the mean \pm s.e.m. For electrophysiological and behavioral tests, ANOVA followed by Dunnett's multiple comparisons post-hoc was used to analyze the data (GraphPad Prism5). The number of oocytes, neurons, or animals per group is indicated in the figure legend. The statistical values (F, degrees of freedom) can be found in the results section. Student's $t$-test was used to analyze the immunoprecipitation data

\section{RESULTS \\ SELECTION OF MISSENSE SNPS IN CHRNB4 FOR FUNCTIONAL ANALYSIS}

We searched the SNP database for missense polymorphisms in the coding region (cSNPs) of the CHRNB4 gene (http://www.ncbi. nlm.nih.gov/projects/SNP/snpref.cgi?geneId=1143). The database reports 67 missense variants with very low or not determined minor allele frequency (MAF). Given that validation studies have been reported for only a third of these missense variants, and that informative SNPs need to be polymorphic on the two different alleles (Li et al., 2007), we selected 14 variants that had either an heterozygosity index $p \geq 0.004$, or were validated by frequency or genotype data (minor alleles observed in at least two chromosomes) (Table 1). We introduced the corresponding mutations in the mouse Chrnb4 gene by site-directed mutagenesis of the indicated nucleotides in the mouse sequence (Table 1). It is interesting to note that 8 of the 14 selected SNPs result in a change in the charge or hydrophobicity of the aminoacid residue and that 6 of these SNPs correspond to mutations of arginine (R) residues (positively charged) (Table 1). Given that all of these variants are

Table 1 | Missense SNPs in CHRNB4 and corresponding variants introduced in the mouse sequence.

\begin{tabular}{|c|c|c|c|c|c|c|c|}
\hline rs number & MAF & HET & ${ }^{\mathrm{h}}$ Missense & ${ }^{m}$ Missense & ${ }^{m}$ Mut & Aa charge & SIFT \\
\hline 72648898 & ND & 0.004 & R39C & R38C & TGC & + to $O$ & DAMAGING \\
\hline 12914008 & 0.0165 & 0.062 & T91I & A90I & ATC & $\mathrm{H}$ to $\mathrm{H}$ & DAMAGING \\
\hline 149832833 & 0.0005 & 0.001 & $\mathrm{R} 109 \mathrm{H}$ & $\mathrm{R} 108 \mathrm{H}$ & CAC & + to + & TOLERATED \\
\hline 56095004 & 0.0037 & 0.012 & $\mathrm{R} 136 \mathrm{Q}$ & $\mathrm{R} 135 \mathrm{Q}$ & CAG & + to $O$ & TOLERATED \\
\hline 79914661 & ND & 0.02 & R322S & R321S & AGT & + to $O$ & DAMAGING \\
\hline 56235003 & 0.0051 & 0.014 & R349C & R348C & TGC & + to $O$ & DAMAGING \\
\hline 61737499 & 0.0005 & 0.016 & T375I & T374I & ATC & $\mathrm{O}$ to $\mathrm{H}$ & TOLERATED \\
\hline 75124790 & ND & 0.129 & N391T & N394T & ACC & $\mathrm{O}$ to $\mathrm{O}$ & TOLERATED \\
\hline 56317523 & 0.0018 & 0.004 & A435V & A348V & GTA & $\mathrm{H}$ to $\mathrm{H}$ & DAMAGING \\
\hline
\end{tabular}

Reference ID numbers (rs number), validated minor allele frequencies (MAF) and heterozygosity rates (HET) of the SNPS in the coding sequence of CHRNB4 that cause non-synonymous missense mutations in human carriers ( ${ }^{h}$ missense). The corresponding missense variants were introduced in the mouse sequence $\left({ }^{m}\right.$ missense) with the indicated nucleotide substitutions ( ${ }^{m}$ mut) in bold. In most cases the missense variant changed the charge of the aminoacid (Aa) (indicated as + , positively charged; O, polar no charge; - negatively charged; $H$, hydrophobic). The last column indicates the SIFT (Sorting Intolerant from Tolerant) value predicts the impact of a residue substitution on protein function (http://siftdna.org/www/Extended_SIFT_chr_coords_submit.html). 
in the extracellular domain and intracellular loop of the $\beta 4$ subunit (Figure 1A), this suggests that some of these variants may alter the hydrophobicity or electrostatic charge of these functional domains in the $\beta 4$ subunit.

\section{NICOTINE-ELICITED CURRENTS OF $\alpha 3 \beta 4$ nAChR AND MISSENSE B4 VARIANTS}

To begin to assess the influence of the selected missense $\beta 4$ variants on nicotine-mediated currents, we performed two electrode voltage clamp recordings in Xenopus laevis oocytes. Oocytes were co-injected with equal amounts of transcripts encoding the $\alpha 3 \mathrm{nAChR}$ subunit and wild-type (wt) $\beta 4$ or $\alpha 3$ and each of the $\beta 4$ point mutants ( $1 \mathrm{ng}$ of $\alpha$ and $1 \mathrm{ng}$ of $\beta$ per oocyte). As shown in Figure 1, nicotine-elicited currents were observed for all the selected missense $\beta 4$ variants except for the N394T and F463Y variants that failed to express at the oocyte surface. We observed that two variants (red bars, Figure 1) significantly differed from $\alpha 3 \beta 4$ control currents. These were the $\beta 4$ R348C variant (corresponding to the human $\mathrm{R} 349 \mathrm{C}$ ) which showed significantly reduced currents $(0.12 \pm 0.43$ normalized amplitude increase) in agreement with previous reports (Moriconi et al., 2011; Richards et al., 2011), and the D447Y variant (corresponding to the human $\mathrm{D} 444 \mathrm{Y})$ that showed significantly larger currents $(3.13 \pm 0.24$ normalized amplitude increase). This rare SNP reported by Weiss and colleagues (Weiss et al., 2008) has not been previously characterized functionally. The 8 variants indicated in black bars (Figure 1) exhibited currents that did not differ significantly from control oocytes injected with $\alpha 3 \beta 4 w t$ (average current amplitude for a3b4 wt $0.88 \pm 0.20 \mu \mathrm{A}$ ) and were not further analyzed. The variants A90I (human T91I variant) and T374I (human T375I), showed slightly higher currents (blue bars in Figure 1) that were not significantly different from wt. However, since these 2 variants are the only SNPs in CHNRB4 that have been associated with nicotine dependence (Haller et al., 2012), we sought to further characterize them in neurons. Based on these results and criteria we selected the variants A90I, R348C, T374I, and D447Y of the $\beta 4$ subunit for further analysis.

\section{$\beta 4$ MISSENSE VARIANTS MAINTAIN THE $\beta 4$-RATE-LIMITING PROPERTY}

We previously showed that $\beta 4$ is rate-limiting for $\alpha 3 \beta 4$ activity as it has the capability to increase the density of $\alpha 3 \beta 4$ receptors at the plasma membrane, as well as to potentiate $\alpha 3 \beta 4$ currents when overexpressed in vitro and in a transgenic mouse model (Frahm et al., 2011). The ability of $\beta 4$ to enhance nicotine-evoked currents depends on a unique single residue $\mathrm{S} 435$, which does not exist in other $\beta$ nAChR subunits and can confer this capability to $\beta 2$ when introduced in the corresponding arginine residue (Frahm et al., 2011).

To evaluate whether this unique property of $\beta 4$ was conserved in the four selected $\beta 4$ point mutants we next tested these point variants in overexpression conditions. Oocytes were injected with equal amounts of $\alpha 3$ and $\beta 4$ transcripts (1:1 ratio) and compared to oocytes injected with 10 times more of the $\beta 4$ transcript than of $\alpha 3$ (1:10 ratio). Representative traces of these recordings are shown in Figure 2A. It is interesting to note that the $\beta 4 \mathrm{R} 348 \mathrm{C}$ mutant not only leads to reduced agonist-induced currents, but also has slower kinetics of decay during ligand application (Figure 2A), consistent with reported electrophysiological recordings in cells (Moriconi et al., 2011). Upon overexpression, as previously observed for the native $\beta 4$ (Frahm et al., 2011), nicotine-evoked currents increased $6.54 \pm 0.43$ fold for native $\beta 4,6.12 \pm 0.33$ fold for $\beta 4$ A90I, $5.85 \pm 0.68$ fold for $\beta 4$ T374I, and $6.85 \pm 0.07$ fold for $\beta 4 \mathrm{D} 447 \mathrm{Y}$ (Figure 2B). The $\beta 4 \mathrm{R} 348 \mathrm{C}$ variant, which had lower currents at the $1: 1$ ratio $(0.30 \pm 0.030$ fold amplitude normalized to $\alpha 3 \beta 4 \mathrm{wt}$, also showed increased currents reaching $2.97 \pm 0.30$ fold increase upon 1:10 overexpression (Figure 2B).

\section{LENTIVIRAL CONSTRUCTS FOR TARGETED EXPRESSION OF $\alpha 3 \beta 4$ nAChRs IN NEURONS}

In order to analyze $\alpha 3 \beta 4 \mathrm{nAChR}$ currents in neurons and in mice, and evaluate the influence of the $\beta 4$ missense variants, we constructed lentiviral vectors. To avoid potential problems with trafficking or assembly, we designed bicistronic constructs instead of generating fluorescent fusion proteins. The pFU- $\beta 4$ IRES-EGFP construct contains the mouse $\beta 4$ subunit (native or variant) followed by an internal ribosome entry site (IRES) and EGFP to monitor the level of infection, while pFU- $\alpha 3$-IRESmCherry was designed to express the mouse $\alpha 3$ subunit and mCherry (Figure 3). As a control, we used pFU-PE, a lentivirus encoding the transmembrane domain of the PDGF receptor fused to EGFP (PE) (Auer et al., 2010). Prior to functional assays, the infection capabilities and reporter gene expression of the generated lentiviral particles were assayed in cultured rat hippocampal neurons. Bright direct fluorescence was observed in the soma and dendrites of neurons transduced with the $\alpha 3$ and $\beta 4$ viruses (Figure 3).

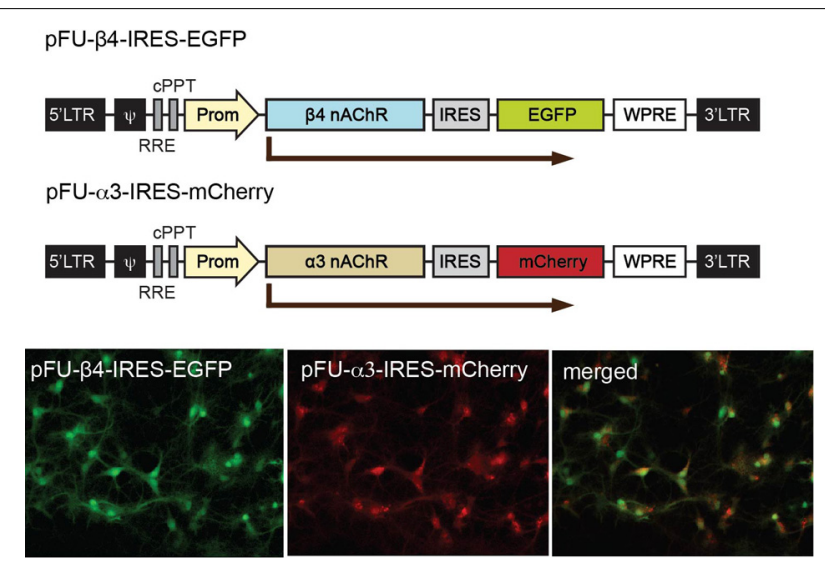

FIGURE 3 | Schematic representation of the viral constructs for expression of $\alpha \mathbf{3}$ and $\beta \mathbf{4} \mathrm{nAChRs}$ in neurons and for stereotactic injections in the Mhb. Graphic representation of the lentiviral vectors encoding $\beta 4$ (pFU- $\beta 4-I R E S-E G F P)$ and $\alpha 3$ (pFU- $\alpha 3-I R E S-m C h e r r y)$. The modules for viral expression are indicated: $\Psi$, packaging sequence; RRE, rev-responsive element; cPPT, central polypurine tract; Prom, human ubiquitin 2 promoter; IRES, internal ribosome entry site sequence; WPRE, woodchuck hepatitis B virus post-transcriptional regulatory element. Photomicrographs of direct fluorescence signal in rat hippocampal neurons transduced with the indicated $\beta 4$ and $\alpha 3$ lentiviruses. 


\section{ELECTROPHYSIOLOGICAL RECORDINGS OF $\beta 4$ MISSENSE VARIANTS IN NEURONS}

To further evaluate the modulatory effect of $\beta 4$ variants on the response of neurons to nicotine, we performed patch clamp analyses in cultured rat hippocampal neurons co-transduced with lentivirus for $\alpha 3$ and $\beta 4$ or $\beta 4$ variants. Representative $\alpha 3 \beta 4 \mathrm{nAChR}$ currents recorded in co-transduced neurons upon pressure application of $30 \mu \mathrm{M}$ nicotine are shown in Figure 4A. Quantitation of average current amplitudes revealed that the $\beta 4$ point mutants A90I, T374I, and D447Y exhibited significantly larger responses to nicotine and that the $\mathrm{R} 348 \mathrm{C}$ variant led to reduced nicotine-evoked current amplitudes, when compared to native $\alpha 3 \beta 4$ responses (Figure 4B). These results confirmed our electrophysiological studies in oocytes (Figure 1) and whole-cell recordings in transfected cells (Moriconi et al., 2011; Haller et al., 2012).

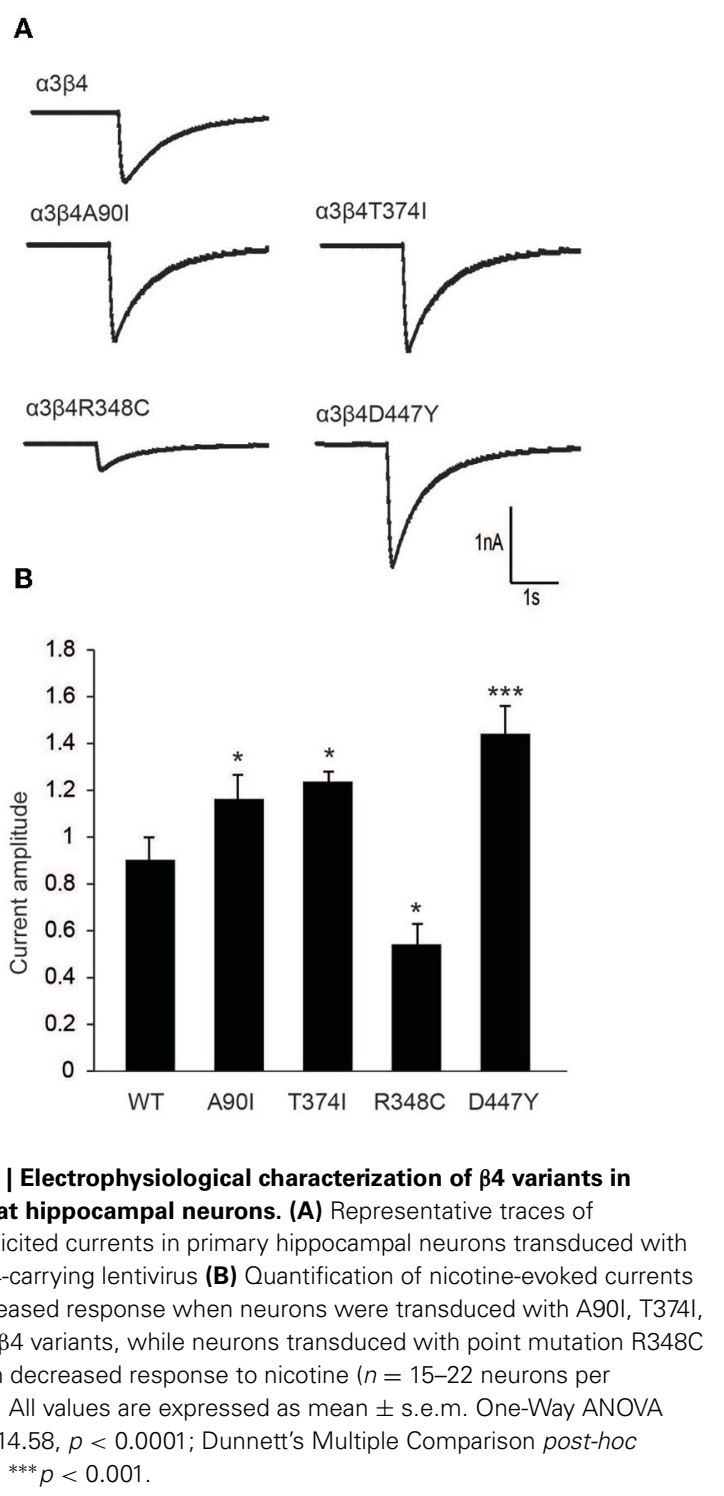

\section{In vivo LENTIVIRAL OVEREXPRESSION OF $\beta 4$ RECONSTITUTES INCREASED $\alpha 3 \beta 4^{*}$ IN THE MHb OF MICE}

We have recently shown that transgenic Tabac mice expressing elevated levels of Chrnb4 at endogenous sites, including the $\mathrm{MHb}$, had increased cytisine resistant- ${ }^{125}$ I-epibatadine ligand binding sites and increased $\alpha 3 \beta 4^{*} \mathrm{nAChR}$ currents, demonstrating that $\beta 4$ has the ability to upregulate the number of $\alpha 3 \beta 4^{*}$ receptors (Frahm et al., 2011). We wanted to assess whether selective overexpression of $\beta 4$ only in the MHb would be able to reproduce this $\alpha 3 \beta 4$ upregulation in vivo. For this reason mice were sterotactically injected bilaterally in the $\mathrm{MHb}$ with either the $\beta 4$ containing lentivirus or the $\mathrm{pFU}-\mathrm{PE}$ control virus (Figure 5A). Quantification of $\alpha 3, \beta 4$, and $\beta 2$ subunits was performed by immunoprecipitation assays from $2 \%$ Triton X-100 habenula
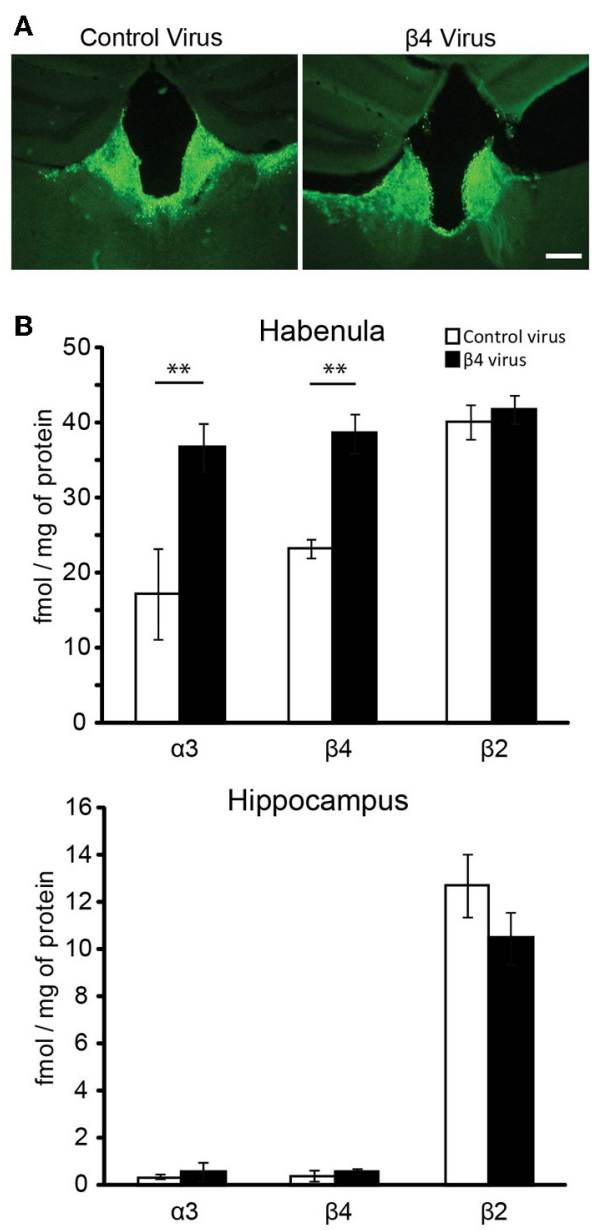

FIGURE 5 | Lentiviral mediated expression of $\beta 4$ in the Mhb and quantification by immunoprecipitation. (A) Representative coronal brain sections of mice injected with pFU-PE control virus or with the $\beta 4$ containing virus pFU- $\beta 4-I R E S-E G F P$. (B) Quantitative immunoprecipitation, expressed as $\mathrm{fmol} / \mathrm{mg}$ of protein of the solubilized [ ${ }^{125} \mathrm{I}$ ]epibatidine-labeled receptors immunoprecipitated by the anti- $\alpha 3,-\beta 4$, or $-\beta 2$ subunit specific antibodies (Grady et al., 2009). The solubilized [ ${ }^{125}$ I]epibatidine-labeled receptors were obtained from the habenula and hippocampus of C57BL/6 mice injected with either control virus or virus carrying the $\beta 4 \mathrm{nAChR}$ subunit ( $n=10$ mice per group). ${ }^{* *} p<0.01$, Student's $t$-test. 
extracts obtained after solubilization of the membranes obtained from the habenula region of injected mice (Figure 5B). The hippocampus was used as an uninfected control region (Figure 5C). The indicated $\alpha 3, \beta 4$, and $\beta 2$ values correspond to the femtomoles of ${ }^{125} \mathrm{I}$-epibatidine labeled receptors immunoprecipitated per $\mathrm{mg}$ of protein. As shown in Figure 5B, there is a very pronounced increase in $\alpha 3$ and $\beta 4$-containing receptors, but no change in the level of $\beta 2$ receptors, in the habenula of mice injected with the $\beta 4$ lentivirus. This suggests that, in the habenula, the $\beta 2$ subunit is not coassembled in receptors containing the $\beta 4$ subunit. The immunoprecipitations from hippocampus of mice injected in the $\mathrm{MHb}$ clearly indicate that there is no change in $\alpha 3 \beta 4$-containing and in $\beta 2$-containing receptors, as expected from the restricted lentiviral-mediated expression of $\beta 4$ by stereotactic injection. These results demonstrate that $\beta 4$, as the rate-limiting subunit for assembly, can increase $\alpha 3$ incorporation and subsequent surface expression.

\section{LENTIVIRAL-MEDIATED EXPRESSION OF MISSENSE $\beta 4$ VARIANTS IN THE MHb ALTERS NICOTINE CONSUMPTION IN MICE}

Given that lentiviral expression of only $\beta 4$ is sufficient to upregulate $\alpha 3 \beta 4$ receptors in vivo in the habenula (Figure 5), and that transgenic Tabac mice expressing elevated levels of Chrnb4 at endogenous sites, including the $\mathrm{MHb}$, consume less nicotine (Frahm et al., 2011), we wanted to confirm the critical role of the $\mathrm{MHb}$ in nicotine consumption by employing lentiviralmediated transduction to overexpress $\beta 4$ in this particular brain region. We measured nicotine consumption using the two-bottle drinking test. Because nicotine solutions have bitter taste, control experiments were performed with a bitter solution (quinine). These measurements did not show significant differences in consumption between wt and Tabac mice (Frahm et al., 2011). We determined the relative volume of drank nicotine (diluted in $2 \%$ saccharin) compared to $2 \%$ saccharine sweetened water in $\mathrm{wt}$, Tabac mice and wt mice injected with either a control virus, or virus encoding $\beta 4$ or the $\beta 4$ variants T374I and R348C. As shown in Figure 6, lentiviral-mediated overexpression of $\beta 4$ in the $\mathrm{MHb}$ of injected wild-type mice is sufficient to cause a strong aversion to $50 \mu \mathrm{g} / \mathrm{ml}$ nicotine in a two-bottle choice paradigm ( $\beta 4$ injected mice: $36.58 \pm 5.8 \%, p<0.05)$ compared to mice injected with the PE control virus $(51.35 \pm 4.2 \%, p<0.05)$. The nicotine aversion observed in wt mice injected with $\beta 4$ lentivirus is less pronounced than the one observed in transgenic Tabac mice $(11.86 \pm 2.90 \%$ nicotine, $p<0.05)$, most likely because of higher expression of $\beta 4$ achieved in Tabac transgenic mice [which contain more than 20 copies of the BAC encoding Chrnb4-a3-EGFP, (Frahm et al., 2011)] than in mice injected with the $\beta 4$-lentivirus (Figure 6B). Mice injected with the lentivirus encoding the $\beta 4$ T374I gain-of-function variant also showed reduced nicotine intake $(39.54 \pm 3.6 \%, p<0.05)$, while mice expressing the lossof-function $\beta 4 \mathrm{R} 349 \mathrm{C}$ variant showed no alteration in nicotine consumption $(51.15 \pm 8.4 \%, p<0.05)$ with respect to the mice injected with the PE control virus (Figure 6B). Thus, our studies support a protective nature for the $\beta 4 \mathrm{~T} 374 \mathrm{I}$ variant in the animal model, in agreement with genome-wide association studies that linked the corresponding SNP T375I in CHRNB4 to decreased risk for nicotine addiction in humans (Haller et al., 2012).

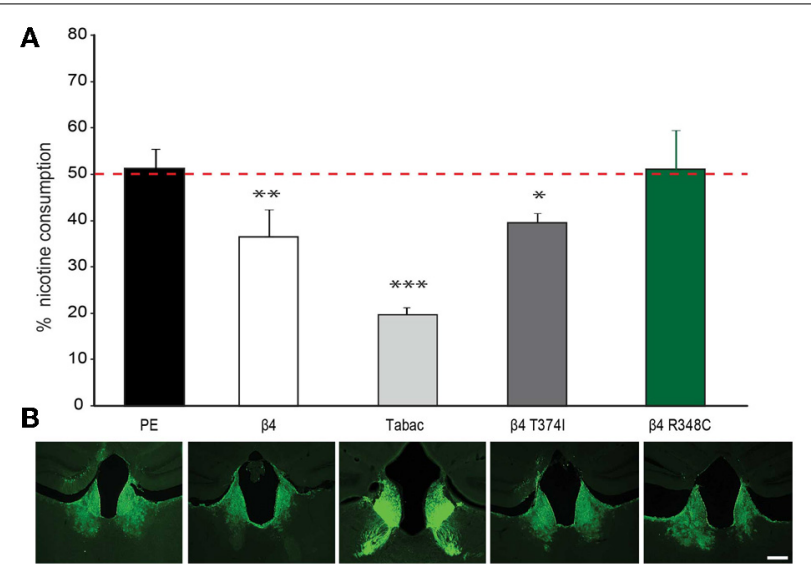

FIGURE 6 | Nicotine intake in mice injected in the Mhb with $\beta 4$ and $\beta 4$ missense variants. (A) Two-bottle choice nicotine consumption in Tabac mice and in $\mathrm{C} 57 \mathrm{BL} / 6$ mice after stereotactic, bilateral $\mathrm{MHb}$ injection of lentivirus carrying control (PE), wild-type $\beta 4$ or $\beta 4$ containing the R348C or T374I mutations constructs ( $n=6-8$ mice per group). All values are expressed as mean \pm s.e.m. One-Way ANOVA $F_{(4,29)}=25.25$, $p<0.0001$; Dunnett's Multiple Comparison post-hoc $* p<0.05,{ }^{* *} p<0.01$, ${ }^{* * *} p<0.001$. (B) Representative images of coronal brain sections showing the site of lentivirus injection in $\mathrm{MHb}$ for $\mathrm{PE}, \beta 4 \mathrm{~T} 374 \mathrm{I}$, and $\beta 4 \mathrm{R} 348 \mathrm{C}$ in $\mathrm{C} 57 \mathrm{BL} / 6$ mice or eGFP expression in the $\mathrm{MHb}$ and fasciculus retroflexus in Tabac transgenic mice.

\section{DISCUSSION}

Chronic exposure to nicotine, the highly addictive component of tobacco, is thought to alter the neural circuits to promote changes that sustain the use of cigarettes (Paolini and De Biasi, 2011). The CHRNA5-A3-B4 gene cluster encoding the $\alpha 5, \alpha 3$, and $\beta 4$ and subunits of the nAChR has been linked with heavy tobacco use and a high risk of relapse (Saccone et al., 2009). The studies presented here establish that the $\beta 4$ nicotinic receptor subunit regulates nicotine intake in mice, that restricted expression of $\beta 4$ in the $\mathrm{MHb}$ is sufficient to produce nicotine aversion, and that four residues corresponding to CHRNB4 SNP variants found in humans, A90I, T375I, R349C, and D444Y, have profound effects on nicotine currents and nicotine consumption in mice.

Three main conclusions can be drawn from these studies. First, $\beta 4$ is rate-limiting in the formation of $\alpha 3 \beta 4^{*}$ nAChRs. Hence when $\beta 4$ is overexpressed, either in transgenic Tabac mice, carrying several copies of a transgene of the Chrnb4-Chrna3GFP-Chrna5 gene cluster, or by lentivirus-mediated transduction, the presence of supplementary $\beta 4$ subunits favors the recruitment of $\alpha 3$ into additional $\alpha 3 \beta 4$ receptors. This $\beta 4$-mediated upregulation of $\alpha 3 \beta 4 \mathrm{nAChRs}$ detected by ligand binding, electrophysiological and immunoprecipitation assays indicates that these additional $\alpha 3 \beta 4$ receptors are at the plasma membrane. This is important to consider in the context of $\mathrm{nAChR}$ dynamics and trafficking and the effect of nicotine on receptor upregulation. For instance, it has been shown that nicotine enhances pentamer assembly of the $\alpha 3 \beta 4 \mathrm{nAChR}$ combination (Mazzo et al., 2013). Since unassembled subunits are rapidly degraded by the endoplasmic-reticulum-associated protein degradation 
process (Christianson and Green, 2004), and efficient assembly of this nAChR subtype has been reported (Wang et al., 1998; Sallette et al., 2004), it is very plausible that increasing the number of available $\beta 4$ subunits increases assembly and contributes to the $\alpha 3 \beta 4$ receptor upregulation that we have observed in Tabac mice and $\beta 4$-lentivirus injected mice. Consistent with this hypothesis, recent studies have shown that $\alpha 3 \beta 4$ pentamers with three instead of two $\beta 4$ subunits are less prone to degradation. This $\beta 4$ protective effect depends on the endoplasmic reticulum export motif present in $\beta 4{ }_{345}(\mathrm{LFM})_{347}$ which is lacking in $\alpha 3$ (Mazzo et al., 2013). Interestingly, this motif lies at one residue distance from the loss-of-function R349C variant that we have identified in our functional assays. Consistently, this mutant shows decreased currents and reduced membrane insertion (Moriconi et al., 2011; Richards et al., 2011). Hence these studies on $\beta 4$ variants, together with our previous work showing that the native $\beta 4 S 435$ residue has the unique capability to confer the rate limiting capability of $\beta 4$ to other beta $\mathrm{nAChR}$ subunits, strengthens the view that the $\beta 4$ subunit has a distinctive role in $\mathrm{nAChR}$ trafficking and assembly. The fact that this subunit has recently been linked to nicotine dependence further validates its key role in mediating nicotine responses.

Second, this study provides a novel paradigm to test the influence of $\beta 4$ variants in nicotine consumption in vivo. Because of the unique capability of $\beta 4$ to upregulate $\alpha 3 \beta 4 \mathrm{nAChR}$ activity, and because the $\mathrm{MHb}$ is such a small brain structure highly enriched in $\alpha 3 \beta 4$ receptors, the possibility to test the behavioral outcome of rare missense $\beta 4$ variants in this system is valuable. The two SNPs analyzed here, A90I in the extracellular domain and T374I in the intracellular loop, have been linked to a reduced risk of nicotine dependence in GWAS and shown to increase nicotine currents in transfected HEK293 cells (Haller et al., 2012). Consistently, our screen of $\beta 4$ missense variants identified these two SNPs, and an additional unstudied SNP, D447Y, as the variants with significantly higher nicotine-elicited currents in oocytes and in mammalian neurons. Subsequent viral-mediated expression of one of these gain-of-function alleles in the $\mathrm{MHb}$ resulted in strong aversion for nicotine, in support of the decreased risk of developing nicotine dependence in individuals carrying this allele. We also identified a variant with $70 \%$ less currents than native $\beta 4$. This missense mutant, $\beta 4 \mathrm{R} 348 \mathrm{C}$, has not yet been identified in GWAS of nicotine dependence, but has been linked to sALS (Sabatelli et al., 2009; Moriconi et al., 2011). Cellular studies have shown that the $\beta 4 \mathrm{R} 349 \mathrm{C}$ mutation, independent of the companion $\alpha$ subunit, causes a reduction in potency of both ACh and nicotine, decreases the density of whole-cell current evoked by maximal transmitter concentrations, and alters the kinetics of ACh-evoked whole-cell currents (Moriconi et al., 2011; Richards et al., 2011). Interestingly overexpression of this loss-of-function mutant in the habenula fails to induce nicotine aversion in mice in comparison to mice injected with native $\beta 4$ or gain-of-function $\beta 4$ variants. It is important to note that wild-type $\beta 4$ and the $\beta 4348 \mathrm{C}$ mutant contain their native $\mathrm{S} 435$ residue, which is able to increase currents upon overexpression (Figure 2). Hence lentiviral overexpression of $\beta 4348 \mathrm{C}$ will produce more functional $\alpha 3 \beta 4$ receptors and subsequently higher nicotine mediated currents than if this mutant would be expressed in a knock-in situation, comparable to a $\beta 4 \mathrm{R} 348 \mathrm{C}$ human carrier. Of course, the use of viral vectors has some limitations. Most notable are the facts that viral transduction results in overexpression of the carried gene, and that expression can sometimes occur in adjacent brain areas. While these points need to be considered, the facts that we have observed phenotypes consistent with the properties of the mutant receptors documented in oocytes and hippocampal neurons, and that very little expression of $\mathrm{nAChR}$ alpha subunits is detected in the surrounding cell types (Allen Brain Atlas images in supplementary material) that can combine with $\beta 4$ to form functional receptors, support the conclusions drawn based on our viral expression studies. Another consideration to acknowledge is that the two-bottle free-choice drinking test paradigm used here measures aversion but not preference, and that mice injected with the non- $\beta 4$ carrying control virus consumed a similar nicotine volume as mice overexpressing the $\beta 4 \mathrm{R} 348 \mathrm{C}$. Based on these considerations we can infer that if one single allele of this mutant was present, mice would have shown preference for nicotine. Thus, it would be interesting to assess whether sALS patients carrying this mutation are more prone to develop smoking dependence. From these data we can conclude that increased $\beta 4$-mediated $\mathrm{nAChR}$ currents increase aversion to nicotine, while decreased currents decrease aversion, in favor of the hypothesis that the MHb-IPN pathway limits consumption of high doses of nicotine. Hence these results are relevant in the context of MHb-mediated control of nicotine consumption and the possibility to employ $\beta 4$ to enhance or depress habenular activity.

Third, it is important to consider the fact that missense variants of $\beta 4$ are so rare in the human population. For instance, the $\beta 4 \mathrm{R} 348 \mathrm{C}$ loss-of-function variant that decreases $\alpha 3 \beta 4^{*}$ currents to a similar extent as the $\alpha 5 \mathrm{D} 398 \mathrm{~N}$ variant, and could potentially be linked to nicotine dependence, is present only in $1.4 \%$ of the human population (Table 1), in stark contrast with the rs16969968 corresponding to $\alpha 5 \mathrm{D} 398 \mathrm{~N}$ present in $28.9 \%$ of smokers (http://www.ncbi.nlm.nih.gov/projects/SNP/snpref. cgi? $r s=16969968)$. Given that the $\beta 4 R 348 \mathrm{C}$ mutation has been associated with sALS and that $\beta 4$ is present in ganglionic and motor neurons, it is tempting to hypothesize that mutations in $\beta 4$ would be too damaging to be carried to the progeny. The fact that smoking may enhance sALS (Armon, 2009) may underlie the importance of $\alpha 3 \beta 4^{*}$ activity in enhancing disease susceptibility, in particular in association with ambient causes such as cigarette smoking. Hence very few studies have found significant associations of CHRNB4 itself with smoking phenotypes, perhaps because it is the rate-limiting subunit when assembling $\beta 4^{*} \mathrm{nAChRs}$, suggesting that mutations in this subunit are less tolerated and thus found less often in the population.

\section{AUTHOR CONTRIBUTIONS}

Inés Ibañez-Tallon designed research; Marta A. Ślimak, Jessica L. Ables, Silke Frahm, Beatriz Antolin-Fontes, Milena Moretti, and Cecilia Gotti performed research; Cecilia Gotti and Inés IbañezTallon analyzed data; Inés Ibañez-Tallon and Jessica L. Ables wrote the paper. 


\section{ACKNOWLEDGMENTS}

The authors thank Sebastian Auer and Monika Schwarz for their technical assistance; This work was supported by the NINDS grant 4R00NS067239-03 (Jessica L. Ables), the Italian PRIN 2009R7WCZS, the European Union grant Eranet and the CNR Research Project on Aging (Cecilia Gotti) and the Helmholtz Association 31-002 and Sonderforschungsbereich SFB665 (Inés Ibañez-Tallon).

\section{SUPPLEMENTARY MATERIAL}

The Supplementary Material for this article can be found online at: http://www.frontiersin.org/journal/10.3389/fnhum. 2014.00012/abstract

\section{REFERENCES}

Armon, C. (2009). Smoking may be considered an established risk factor for sporadic ALS. Neurology 73, 1693-1698. doi: 10.1212/WNL.0b013e3181cldf48

Auer, S., Sturzebecher, A. S., Juttner, R., Santos-Torres, J., Hanack, C., Frahm, S., et al. (2010). Silencing neurotransmission with membrane-tethered toxins. Nat. Methods 7, 229-236. doi: 10.1038/nmeth.1425

Bierut, L. J., Madden, P. A., Breslau, N., Johnson, E. O., Hatsukami, D., Pomerleau, O. F., et al. (2007). Novel genes identified in a high-density genome wide association study for nicotine dependence. Hum. Mol. Genet. 16, 24-35. doi: 10.1093/hmg/ddl441

Butt, C. M., King, N. M., Hutton, S. R., Collins, A. C., Stitzel, J. A. (2005). Modulation of nicotine but not ethanol preference by the mouse Chrna4 A529T polymorphism. Behav. Neurosci. 119, 26-37. doi: 10.1037/0735-7044.119.1.26

Christianson, J. C., Green, W. N. (2004). Regulation of nicotinic receptor expression by the ubiquitin-proteasome system. EMBO J. 23, 4156-4165. doi: 10.1038/sj.emboj.7600436

Dittgen, T., Nimmerjahn, A., Komai, S., Licznerski, P., Waters, J., Margrie, T. W., et al. (2004). Lentivirus-based genetic manipulations of cortical neurons and their optical and electrophysiological monitoring in vivo. Proc. Natl. Acad. Sci. U.S.A. 101, 18206-18211. doi: 10.1073/pnas.0407976101

Fowler, C. D., Lu, Q., Johnson, P. M., Marks, M. J., and Kenny, P. J. (2011). Habenular alpha5 nicotinic receptor subunit signalling controls nicotine intake. Nature 471, 597-601. doi: 10.1038/nature09797

Frahm, S., Slimak, M. A., Ferrarese, L., Santos-Torres, J., Antolin-Fontes, B., Auer, S., et al. (2011). Aversion to nicotine is regulated by the balanced activity of beta 4 and alpha5 nicotinic receptor subunits in the medial habenula. Neuron 70, 522-535. doi: 10.1016/j.neuron.2011.04.013

Franklin, K. B. J., and Paxinos, G. (2008). The Mouse Brain in Stereotaxic Coordinates, 3rd Edn. San Diego: Academic Press.

Gallego, X., Molas, S., Amador-Arjona, A., Marks, M. J., Robles, N., Murtra, P., et al. (2012). Overexpression of the CHRNA5/A3/B4 genomic cluster in mice increases the sensitivity to nicotine and modifies its reinforcing effects. Amino Acids 43, 897-909. doi: 10.1007/s00726-011-1149-y

Glatt, A. R., Denton, K., and Boughter, J. D. Jr. (2009). Variation in nicotine consumption in inbred mice is not linked to orosensory ability. Chem. Senses 34, 27-35. doi: 10.1093/chemse/bjn049

Gong, S., Zheng, C., Doughty, M. L., Losos, K., Didkovsky, N., Schambra, U. B., et al. (2003). A gene expression atlas of the central nervous system based on bacterial artificial chromosomes. Nature 425, 917-925. doi: 10.1038/nature 02033

Gorlich, A., Antolin-Fontes, B., Ables, J. L., Frahm, S., Slimak, M. A., Dougherty, J. D., et al. (2013). Reexposure to nicotine during withdrawal increases the pacemaking activity of cholinergic habenular neurons. Proc. Natl. Acad. Sci. U.S.A. 110, 17077-17082. doi: 10.1073/pnas.1313103110

Grady, S. R., Moretti, M., Zoli, M., Marks, M. J., Zanardi, A., Pucci, L., et al. (2009). Rodent habenulo-interpeduncular pathway expresses a large variety of uncommon nAChR subtypes, but only the alpha3beta 4 and alpha3beta3beta $4 *$ subtypes mediate acetylcholine release. J. Neurosci. 29, 2272-2282. doi: 10.1523/JNEUROSCI.5121-08.2009

Haller, G., Druley, T., Vallania, F. L., Mitra, R. D., Li, P., Akk, G., et al. (2012). Rare missense variants in CHRNB4 are associated with reduced risk of nicotine dependence. Hum. Mol. Genet. 21, 647-655. doi: 10.1093/hmg/ddr498
Harari, O., Wang, J. C., Bucholz, K., Edenberg, H. J., Heath, A., Martin, N. G., et al. (2012). Pathway analysis of smoking quantity in multiple GWAS identifies cholinergic and sensory pathways. PLoS ONE 7:e50913. doi: 10.1371/journal.pone.0050913

Ibanez-Tallon, I., Wen, H., Miwa, J. M., Xing, J., Tekinay, A. B., Ono, F., et al. (2004). Tethering naturally occurring peptide toxins for cell-autonomous modulation of ion channels and receptors in vivo. Neuron 43, 305-311. doi: 10.1016/j.neuron.2004.07.015

Kapoor, M., Wang, J. C., Bertelsen, S., Bucholz, K., Budde, J. P., Hinrichs, A., et al. (2012). Variants located upstream of CHRNB4 on chromosome 15q25.1 are associated with age at onset of daily smoking and habitual smoking. PLoS ONE 7:e33513. doi: 10.1371/journal.pone.0033513

Li, X., Self, S. G., Galipeau, P. C., Paulson, T. G., and Reid, B. J. (2007). Direct inference of SNP heterozygosity rates and resolution of LOH detection. PLoS Comput. Biol. 3:e244. doi: 10.1371/journal.pcbi.0030244

Liang, Y., Salas, R., Marubio, L., Bercovich, D., De Biasi, M., Beaudet, A. L., et al. (2005). Functional polymorphisms in the human beta4 subunit of nicotinic acetylcholine receptors. Neurogenetics 6, 37-44. doi: 10.1007/s10048004-0199-7

Matta, S. G., Balfour, D. J., Benowitz, N. L., Boyd, R. T., Buccafusco, J. J., Caggiula, A. R., et al. (2007). Guidelines on nicotine dose selection for in vivo research. Psychopharmacology (Berl.) 190, 269-319. doi: 10.1007/s00213-006-0441-0

Mazzo, F., Pistillo, F., Grazioso, G., Clementi, F., Borgese, N., Gotti, C., et al. (2013). Nicotine-modulated subunit stoichiometry affects stability and trafficking of alpha3beta4 nicotinic receptor. J. Neurosci. 33, 12316-12328. doi: 10.1523/JNEUROSCI.2393-13.2013

Meliska, C. J., Bartke, A., Vandergriff, J. L., and Jensen, R. A. (1995). Ethanol and nicotine consumption and preference in transgenic mice overexpressing the bovine growth hormone gene. Pharmacol. Biochem. Behav. 50, 563-570. doi: 10.1016/0091-3057(94)00345-9

Moriconi, C., Di Angelantonio, S., Piccioni, A., Trettel, F., Sabatelli, M., and Grassi, F. (2011). Mutant human beta4 subunit identified in amyotrophic lateral sclerosis patients impairs nicotinic receptor function. Pflugers Arch. 461, 225-233. doi: 10.1007/s00424-010-0905-2

Paolini, M., and De Biasi, M. (2011). Mechanistic insights into nicotine withdrawal. Biochem. Pharmacol. 82, 996-1007. doi: 10.1016/j.bcp.2011.07.075

Richards, C. I., Srinivasan, R., Xiao, C., Mackey, E. D., Miwa, J. M., and Lester HA (2011). Trafficking of alpha $4^{*}$ nicotinic receptors revealed by superecliptic phluorin: effects of a beta4 amyotrophic lateral sclerosis-associated mutation and chronic exposure to nicotine. J. Biol. Chem. 286, 31241-31249. doi: 10.1074/jbc.M111.256024

Robinson, S. F., Marks, M. J., and Collins, A. C. (1996). Inbred mouse strains vary in oral self-selection of nicotine. Psychopharmacology (Berl.) 124, 332-339. doi: 10.1007/BF02247438

Sabatelli, M., Eusebi, F., Al-Chalabi, A., Conte, A., Madia, F., Luigetti, M., et al. (2009). Rare missense variants of neuronal nicotinic acetylcholine receptor altering receptor function are associated with sporadic amyotrophic lateral sclerosis. Hum. Mol. Genet. 18, 3997-4006. doi: 10.1093/hmg/ddp339

Saccone, N. L., Wang, J. C., Breslau, N., Johnson, E. O., Hatsukami, D., Saccone, S. F., et al. (2009). The CHRNA5-CHRNA3-CHRNB4 nicotinic receptor subunit gene cluster affects risk for nicotine dependence in African-Americans and in European-Americans. Cancer Res. 69, 6848-6856. doi: 10.1158/00085472.CAN-09-0786

Saccone, S. F., et al. (2007). Cholinergic nicotinic receptor genes implicated in a nicotine dependence association study targeting 348 candidate genes with 3713 SNPs. Hum. Mol. Genet. 16, 36-49. doi: 10.1093/hmg/ddl438

Salas, R., Sturm, R., Boulter, J., and De Biasi, M. (2009). Nicotinic receptors in the habenulo-interpeduncular system are necessary for nicotine withdrawal in mice. J. Neurosci. 29, 3014-3018. doi: 10.1523/JNEUROSCI. 4934-08.2009

Sallette, J., Bohler, S., Benoit, P., Soudant, M., Pons, S., Le Novere, N., et al. (2004). An extracellular protein microdomain controls up-regulation of neuronal nicotinic acetylcholine receptors by nicotine. J. Biol. Chem. 279, 18767-18775. doi: 10.1074/jbc.M308260200

Sarginson, J. E., Killen, J. D., Lazzeroni, L. C., Fortmann, S. P., Ryan, H. S., Schatzberg, A. F., et al. (2011). Markers in the 15q24 nicotinic receptor subunit gene cluster (CHRNA5-A3-B4) predict severity of nicotine addiction and response to smoking cessation therapy. Am. J. Med. Genet. B Neuropsychiatr. Genet. 156B, 275-284. doi: 10.1002/ajmg.b.31155 
Sturzebecher, A. S., Hu, J., Smith, E. S., Frahm, S., Santos-Torres, J., Kampfrath, B., et al. (2010). An in vivo tethered toxin approach for the cell-autonomous inactivation of voltage-gated sodium channel currents in nociceptors. J. Physiol. 588, 1695-1707. doi: 10.1113/jphysiol.2010.187112

Thorgeirsson, T. E., Geller, F., Sulem, P., Rafnar, T., Wiste, A., Magnusson, K. P., et al. (2008). A variant associated with nicotine dependence, lung cancer and peripheral arterial disease. Nature 452, 638-642. doi: 10.1038/ nature 06846

Wang, F., Nelson, M. E., Kuryatov, A., Olale, F., Cooper, J., Keyser, K., et al. (1998). Chronic nicotine treatment up-regulates human alpha3 beta2 but not alpha3 beta 4 acetylcholine receptors stably transfected in human embryonic kidney cells. J. Biol. Chem. 273, 28721-28732. doi: 10.1074/jbc.273. 44.28721

Weiss, R. B., Baker, T. B., Cannon, D. S., von Niederhausern, A., Dunn, D. M., Matsunami, N., et al. (2008). A candidate gene approach identifies the CHRNA5-A3-B4 region as a risk factor for age-dependent nicotine addiction. PLoS Genet. 4:e1000125. doi: 10.1371/journal.pgen. 1000125
Conflict of Interest Statement: The authors declare that the research was conducted in the absence of any commercial or financial relationships that could be construed as a potential conflict of interest.

Received: 21 October 2013; paper pending published: 04 November 2013; accepted: 07 January 2014; published online: 27 January 2014.

Citation: Ślimak MA, Ables JL, Frahm S, Antolin-Fontes B, Santos-Torres J, Moretti $M$, Gotti C and Ibañez-Tallon I (2014) Habenular expression of rare missense variants of the $\boldsymbol{\beta} 4$ nicotinic receptor subunit alters nicotine consumption. Front. Hum. Neurosci. 8:12. doi: 10.3389/fnhum.2014.00012

This article was submitted to the journal Frontiers in Human Neuroscience. Copyright (c) 2014 Ślimak, Ables, Frahm, Antolin-Fontes, Santos-Torres, Moretti, Gotti and Ibañez-Tallon. This is an open-access article distributed under the terms of the Creative Commons Attribution License (CCBY). The use, distribution or reproduction in other forums is permitted, provided the original author(s) or licensor are credited and that the original publication in this journal is cited, in accordance with accepted academic practice. No use, distribution or reproduction is permitted which does not comply with these terms. 